\title{
Pulsar Magnetospheres: Classical, Quasi-Classical and Quantum Descriptions
}

\author{
A. A. da Costa \\ Centro de Electrodinâmica, Instituto Superior Técnico, 1049-001, \\ Lisboa, Portugal (antonio_da_costa@ieee.org)
}

\begin{abstract}
The plasma motion in pulsar magnetospheres is no longer classical, but quasi-classical, following stochastic trajectories, when random curvature radiation of high energetic gamma-ray photons takes place. This implies an extension to the relativistic kinetic theory of plasmas. But with high energies involved other quantum radiative processes become important, in the context of vacuum (quantum) electrodynamics. The consequences for pulsar radiation mechanisms will be outlined.
\end{abstract}

\section{Introduction}

The classical description of plasma motion in pulsar magnetospheres is unacceptable and has to be substituted by a quasi-classical (q-classical) one, when radiative effects must be taken into account. In this q-classical description trajectories are stochastic, as the radiated electromagnetic (EM) field has to be described by discrete random radiation of high energetic gamma-ray photons. The associated physical processes will be discussed, in the context of Minkowski space-time, followed by a reference to a geometrodynamical formulation, and an extension of the kinetic theory of plasmas to take into account these phenomena.

Radiative processes happen for large values of the Lorentz factor, $\gamma$. When $\gamma B \gtrsim 10^{9} \mathrm{~T}$, then several quantum processes may become important, and will be discussed. An outline of consequences for radiation mechanisms will be made.

\section{Relativistic Plasma Theory}

Pulsar electron-positron magnetospheric plasmas are driven by a dipolar lowfrequency strong EM wave with a high value of the magnetic induction $B$. The dominant parameter is the strength of the EM wave given by $\eta=e A_{0} / m c$ (da Costa \& Kahn 1982; da Costa 1983), where $A_{0}$ is the amplitude of the vector potential. $\eta \sim 10^{16}$ at the surface of the star, for $\mathrm{B} \sim 10^{8} \mathrm{~T}$, and $\eta \sim 10^{6}-10^{10}$ at the speed of light cylinder (SLC) for periods $\mathrm{T}_{\mathrm{p}} \sim 3 s-33 \mathrm{~ms}$.

$\eta$ is related to the density of the electromagnetic energy $\mathrm{w}_{\mathrm{em}} \sim 10^{23} \mathrm{Jm}^{-3}$ for $\mathrm{B} \sim 10^{8} \mathrm{~T}$, and $\mathrm{w}_{\mathrm{em}} \sim 10^{11} \mathrm{Jm}^{-3}$ for $\mathrm{B} \sim 10^{2} \mathrm{~T}$, its value at the $\mathrm{SLC}$ for the Crab pulsar (CP). The Lorentz force and its associated power $P_{\text {Lor }}$ dominates any other interaction in the process, allowing the single particle approach (SPA).

When no radiative effects are present SPA is given by the Lorentz equation of motion. Putting $\tau=\omega t, \mathbf{p}=m c \varpi, \mathbf{E}=\omega A_{0} \mathcal{E}, \mathbf{v}=c v$ and $\mathbf{B}=\mathcal{B} / \mathrm{R}_{\mathrm{L}}$, where 
$\omega$ is the scalar angular velocity of the pulsar, and $R_{L}$ the SLC radius, then

$$
\frac{d \varpi}{d \tau}=\eta(\mathcal{E}+v \times \mathcal{B}) \equiv \eta \mathcal{F}_{\text {Lor }}
$$

The accelerated plasma may reach very high values of $\gamma$, the Lorentz factor. Thus after leaving the neutron star it is never at rest, and therefore the value of $\mathbf{F}_{\text {Lor }}=\mathbf{E}+\mathbf{v} \times \mathbf{B} \neq \mathbf{0}$. Even if $\mathcal{F}_{\text {Lor }}$ is an infinitesimal quantity, of $\mathrm{O}\left(\eta^{-1}\right)$, $\mathbf{F}_{\text {Lor }}$ may be a meaningful quantity, due to the high value of $\eta$.

The plasma radiates curvature radiation. The power radiated $P_{\text {rad }}$ grows

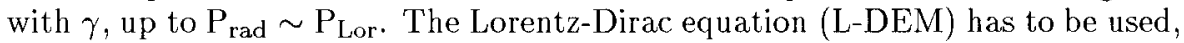
to take into account radiative effects (da Costa \& Kahn 1982; da Costa 1983).

Plasma motion creates charge \& current density distributions (Ch\&CDD), which follow patterns rotating with the angular velocity $\omega$ and change the dipolar nature of the EM field. To identify some phenomena present a simplified model has to be used, with a low density plasma such that the associated charge and current density distributions will not change the dipolar nature of the EM field. We start with the 3 -dimensional (3-D) perpendicular model $(\mathrm{d} . \boldsymbol{\Omega}=\mathbf{0}$, where $\mathrm{d}$ is the dipolar moment, and $\Omega$ is the angular velocity of the pulsar). As this $3-\mathrm{D}$ perpendicular EM field has $\mathbf{E . B} \neq 0$ outside the equatorial plane, we shall use a 2-dimensional equatorial model where $\mathbf{E} \perp \mathbf{B}$, resembling the field of the $3-\mathrm{D}$ equatorial plane (da Costa \& Kahn 1982), with a plasma starting the motion at star surface and sliding along magnetic field lines (MFL). It is shown that for the CP and close to the SLC, along the MFL bent in the forward direction of motion, the particles have so much inertia that they leave MFL. For the CP, particles reach $\gamma \sim 10^{7}$, with a peak frequency $\omega_{0} \sim 10^{23} \mathrm{~Hz}$, hard gamma-ray

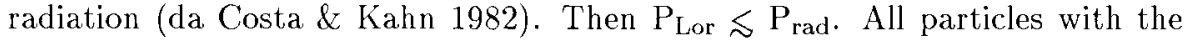
same initial conditions will follow the same trajectory.

L-DEM means that a classical (continuous) radiated field influences in a continuous way the trajectory of the particle. But gamma-ray photons is discontinuous radiation. The radiative reaction correction has to be substituted by a random term, showing radiation of photons at random times, frequency and direction, a markovian process. This is the q-classical regime (da Costa \& Kahn 1997), since particle motion is described by trajectories. Now a stream of particles with the same initial conditions is represented by a bundle of stochastic ones, changing randomly with time, and having a tree-like structure, although reaching the same values of $\gamma \sim 10^{7}$. They will have a spread of energies such that the temperature of an equivalent thermal plasma would be $\mathrm{T}_{\text {eq }} \sim 10^{14} \mathrm{~K}$ reinforcing the idea of a SPA, since thermal effects are negligible. In this regime the energy no longer has a smooth grow but show chaotic behaviour, and this effect together with the tree-like structure of stochastic trajectories show stochastic chaos, so defined because it is a phenomena similar to deterministic chaos, but described by non-linear stochastic equations.

Pulsars are compact stars, with a radius $R_{*} \sim 10 \mathrm{~km}$, implying in geometrodynamic units $\mathrm{R}_{*} / 2 \mathrm{M} \simeq 3$. Therefore geometrodynamics has to be considered, and very important geometrodynamic effects affecting the flow of plasma close to the surface might be expected (Freire \& da Costa 1999). However in such environment q-classical regimes are not markovian (Andrade 1994). This only happens in Minkowski 4-space. But the 4-space curvature at the CP SLC is a perturbation, and therefore the q-classical regime is quasi-markovian there. 


\section{Statistical Behaviour and the Generalized Quantum Connection: the Ginzburg effect}

The description of the plasma behavior as a collective entity, requires the calculation of the kinetic distribution function $f\left(\mathbf{r}_{1}, \mathbf{p}, \mathbf{t}\right)$, where $\mathbf{r}_{1}$ contains the coordinates in the rotation referential. As this calculation demands random frequencies and space discretisation, such description is only possible, provided the latter must be shorter than the minimal dimension of the stochastic phenomena in the direction of motion $\left(10^{-5} R_{L}\right)$, and much smaller than the spread of the trajectories in the plane normal to the average trajectory, with a number of particles in those cells sufficiently significant to identify a plasma. The density necessary to the radiation of gamma-rays is of the order of $10^{12}-10^{14}$ electrons $\mathrm{m}^{-3}$ (da Costa \& Kahn 1982). This implies that each cell must have a minimum of $10^{6}-10^{8}$ particles. Thus it is possible to establish average regular manifolds, formed by trajectories, and to define a statistical kinetic theory, based on the SPA, because of the intense EM fields, and the very large value of $T_{\text {eq }}$.

The magnetospheric stochastic description must be amplified by plasma motion. This effect must be the stronger the weaker the plasma collective description. Q-classical SPA implies stochastic trajectories with a tree like forward structure, and in this way the calculation of $f\left(\mathbf{r}_{1}, \mathbf{p}, t\right)$ will have a continuous recursive character, with multiple bifurcations in all the backward recursive calculation. It is thus possible to face the magnetospheric plasma as a fractal random media, whose genesis explains the incoherent nature of gamma-ray radiation. This plasma characteristic is shared by the associated Ch\&CDD, which might explain radio radiation and therefore the difference among the individual pulses, and the noise observed in some pulsars (Rickett \& al 1975).

Vacuum electrodynamics needs consideration, due to creation of matter and radiation, e.g. the probable interaction of gamma ray radiation with the high energetic plasma. Close to the surface of the star there are hyperstrong magnetic fields close to the classical limit, $\mathrm{B}_{\mathrm{lim}} \sim 4 \times 10^{9} \mathrm{~T}$. For the CP SLC, the product $\gamma B>\mathrm{B}_{\mathrm{lim}}$. The onset of unstable vacuum and associated phenomena must be considered. One of its most interesting possibilities is that the stream of charged particles due to its high energy is sufficiently powerful to break the virtual pairs excited by the hyperstrong electromagnetic field, and therefore to populate the magnetosphere (Fradkin, Gitman \& Shvartzman 1991). This is a sort of magnetic Hawking effect, since it bears strong similarities with the Hawking effect for black holes, changing the plasma distribution functions.

In these extreme conditions other radiative effects might also have to be considered. In a pure classical analysis, transition radiation must also be present since now the vacuum dielectric constant $\epsilon(B)$ (Ginzburg 1989), and the vacuum is a non-homogeneous and anisotropic medium. Therefore the non-linear effects of the combination of curvature and transition radiation may occur, with the presence of transition bremstrahlung and transition scattering (Ginzburg \& Tsytovitch 1990), which add up to the standard curvature radiation effects.

These global phenomena, we call the Ginzburg effect, is particularly relevant close to the surface of the star, since particles approaching the star must lose most of their energy through it. As in this case the vacuum must have a tendency to become highly dense when the particles' $\gamma$ grows, particles leaving the star cannot go beyond $\gamma<10^{3}$ for star surface; and $\gamma<10^{9}$ for the CP SLC. 


\section{Considerations for the future}

Electron-positron plasmas in pulsar magnetospheres show exotic characteristics, provided in curvature radiation $\mathrm{P}_{\text {rad }} \sim \mathrm{P}_{\text {Lor }}$, implying that: the use of SPA is correct, due to the strength of the low frequency EM wave; the plasma radiates high energy gamma-ray photons at random times, frequency and direction; the stream of particles turn into a bundle of stochastic trajectories, with a energy spread such that the equivalent temperature is so large that it reinforces the SPA nature of the phenomena, and its domination over any other.

It is necessary to study a new kinetic theory of radiative plasmas, provided the stochastic nature of the phenomena is dominated by an appropriate plasma density, with length scales smaller than the stochastic SPA, turning the magnetosphere in a random media with fractal nature. This new kinetic theory needs a quantum formulation when $\gamma \mathrm{B} \geq 10^{9} \mathrm{~T}$, when vacuum electrodynamics have to be considered with an associated plasma production, and new quantum radiative effects join the standard curvature radiation ones.

One might expect that these exotic quantum effects will reinforce the characteristics of the SPA, to shape gamma-ray radiation, and of the Ch\&CDD present in the magnetospheres to shape radio frequency radiation and identified in the earlier classical-q-classical regime. However only when we have a complete plasma description we shall be able to construct a realistic radiation mechanism for both pulsar radio frequency and gamma-ray radiation.

Acknowledgments. Most of the present work was produced in close cooperation with the late Professor F. D. Kahn, to whom we dedicate this paper, and whose memory we shall honour forever.

This paper was finished during a sabbatical leave at the Astronomy \& Astrophysics Group of the University of Glasgow, Scotland, United Kingdom.

We thank the Calouste Gulbenkian Foundation, Portugal, for financial support of our presence in this Colloquium.

\section{References}

Andrade, Z. 1994, Pulsar Geometrodynamics: The Structure of the Magnetosphere and the Associated Radiation, Master Thesis in Physics, Instituto Superior Técnico, Lisboa, Portugal

da Costa, A. A. 1983, MNRAS, 204, 1125

da Costa, A. A. \& Kahn F. D. 1982, MNRAS, 199, 211

da Costa, A. A. \& Kahn F. D. 1997, MNRAS, 284, 1

Fradkin, E. S., Gitman, D. M., \& Shvartzman, S. M. 1991, Quantum Electrodynamics with Unstable Vacuum (Berlin: Springer)

Freire, P. C. \& da Costa, A. A. 1999, MNRAS, 304,235

Ginzburg, V.I. 1989, Applications of Electrodynamics in Theoretical Physics and Astrophysics (London: Gordon \& Breach)

Ginzburg, V. I., \& Tsytovitch, V. N. 1990, Transition Radiation and Transition Scattering (London: Adam Hilger)

Rickett, B.J., Hankins, T.H., \& Cordes J. M. 1975, ApJ, 201, 425 